\title{
Использование методов прогнозирования в бюджетном процессе (на примере бюджета Иркутской области)
}

\author{
Клещинский А.И. ${ }^{16}$
}

На сегодняшний день государственный бюджет является одним из важнейших инструментов финансового планирования. От точности его составления зависит эффективность исполнения важных государственных функиий, таких как содержание аппарата управления, внутренняя и внешняя безопасность, сочиальная сфера, поддержка отдельных отраслей хозяйства и пр. Использование методов формализованного прогнозирования в бюджетном прочессе является одним из способов улучшения точности составления бюджета. $B$ статье проведен анализ возможностей применения формализованных методов прогнозирования в бюджетном процессе. На основании результатов анализа предложен алгоритм проведения прогноза в рамках региональных бюджетных систем и дана его оценка на примере бюджета Иркутской области. $B$ результате использование формализованных методов прогнозирования позволяет составлять прогнозы региональных бюджетов с точностью, превыщающей порог в $80 \%$.

\section{JEL: $H 680$}

Ключевые слова: государственный бюджет, формализованные методы прогнозирования, алгоритм проведения прогноза, бюджетный процесс, бюджет Иркутской области, перспективный финансовый план

На сегодняшний день государственный бюджет является одним из важнейших инструментов финансового планирования. От точности его составления зависит эффективность исполнения важных государственных функций, таких как содержание аппарата управления, внутренняя и внешняя безопасность, социальная сфера, поддержка отдельных отраслей хозяйства и пр. Использование методов формализованного прогнозирования в бюджетном процессе является одним из способов улучшения точности составления бюджета. В рамках данной статьи будет проведен анализ возможностей применения формализованных методов прогнозирования в бюджетном процессе, а также предложен алгоритм проведения прогноза в рамках региональных бюджетных систем и дана его оценка на примере бюджета Иркутской области.

Для определения точек приложения методов прогнозирования в рамках бюджетной системы Российской Федерации удобно представить бюджетный процесс в виде следующей схемы:

\footnotetext{
${ }^{16}$ Аспирант Байкальского государственного университета экономики и права.
} 


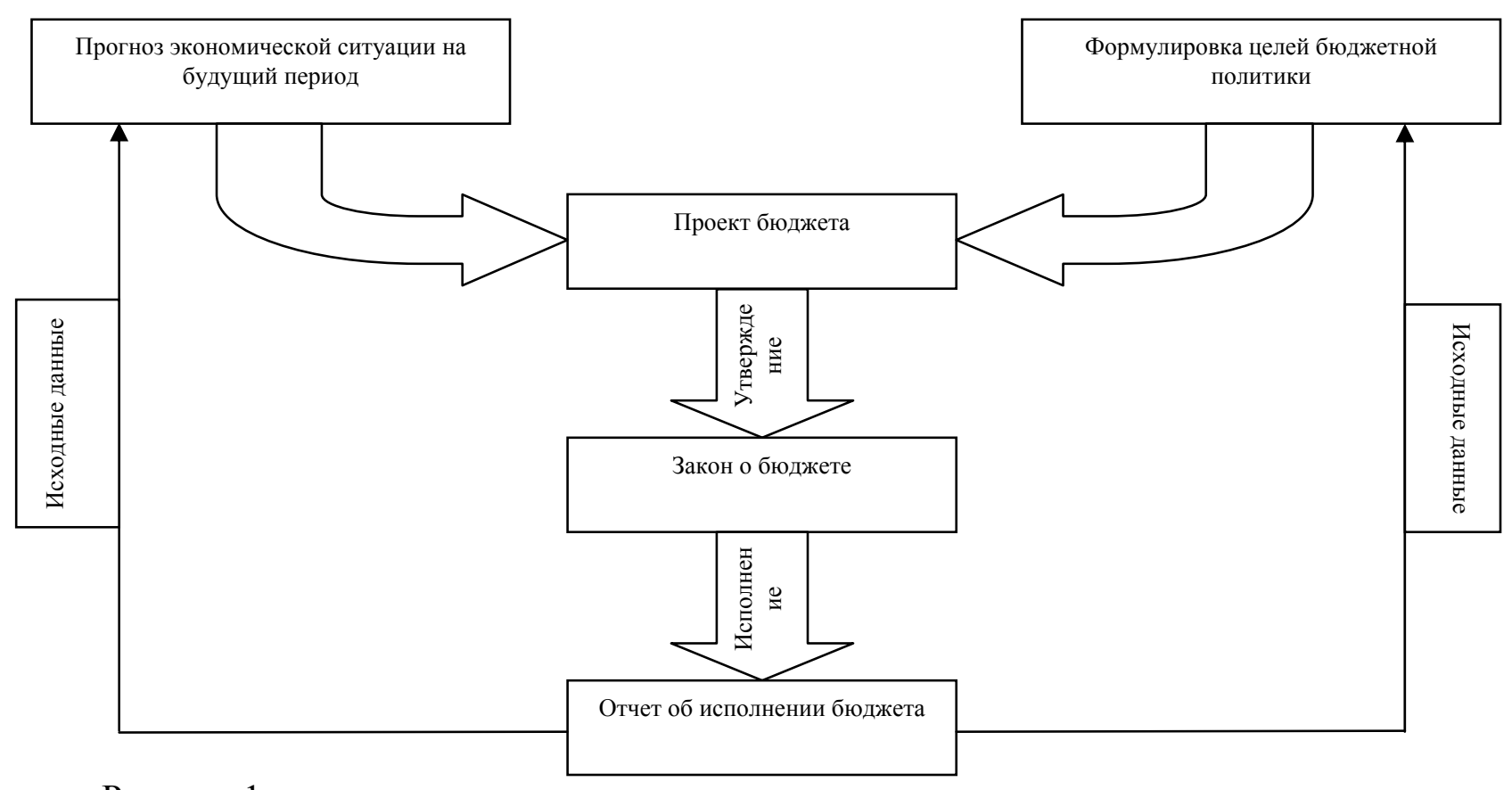

Рисунок 1

В соответствии с представленной схемой, разработка проекта бюджета основывается на прогнозе экономической ситуации на будущий период. Прогноз экономической ситуации может включать как прогнозирование отдельных индикаторов социально-экономического развития, так и разработку прогноза развития финансовой системы в целом (АНО Центр информационных исследований, 2010]). В Российской Федерации документами. представляющими прогнозную информацию, являются прогнозы социально-экономического развития, а также перспективный финансовый план.

В соответствии с Федеральным законом «О государственном прогнозировании и программах социально-экономического развития РФ», прогнозы разрабатываются на кратко, средне- и долгосрочную перспективу (Мысляева, 2007, с. 306-309]). При этом использование категорий методов прогнозирования зависит от типа прогноза.

1. Долгосрочный прогноз - разрабатывается на срок более десяти лет, содержит информацию о прогнозах развития НТП и его влиянии на различных сферы народного хозяйства. При составлении данного прогноза используются преимущественно методы экспертного прогнозирования, так как изменения НТП являются качественными изменениями.

2. Среднесрочный прогноз - разрабатывается на срок от трех до пяти лет, содержит информацию об основных макроэкономических показателей, а также формулировку основных стратегических целей и приоритетов развития страны и регионов. При составлении данного прогноза используются как методы экспертного прогнозирования (в частности, метод построения сценариев), так и математические методы прогнозной экстраполяции.

3. Краткосрочный прогноз - разрабатывается на год, содержит информацию об основных показателях социально-экономического развития страны и регионов. При составлении данного прогноза используется весь потенциал методов прогнозирования.

Перспективный финансовый план формируется на основании среднесрочного прогноза социально-экономического развития и содержит информацию о прогнозных возможностях бюджета. Перспективный финансовый план является инструментом информационной поддержки для законодательных органов власти при принятии управленческих решений и является важным финансовым документом.

Таким образом, разработка проекта бюджета предполагает активное использование разнообразных методов прогнозирования. По мнению автора, в разрезе данной тенденции перспективным направлением является совершенствование подходов в использовании 
математических методов прогнозной экстраполяции для увеличения точности прогнозов. Одним из приложений данного направления может являться совершенствование механизмов прогнозирования показателей перспективного финансового плана. В связи с этим была предложена система прогнозирования отдельных показателей перспективного финансового плана. Разработка системы осуществлялась на основании алгоритма проведения прогноза (Holton Wilson, 2002, c. 50-52):

1. Первым этапом проведения прогноза является определение цели прогнозирования. Поскольку цели создания перспективного финансового плана заложены в Бюджетном кодексе РФ, можно сформулировать цель прогнозирования как создание прогноза показателей перспективного финансового плана для поддержки принятия управленческих решений, связанных с тенденциями развития экономики и социальной сферы.

2. Вторым этапом проведения прогноза является определение объекта прогнозирования. Так как перспективный финансовый план разрабатывается на основании бюджета, то объектом прогнозирования служат бюджетные статьи в разрезе бюджетной классификации Российской Федерации. Принимая во внимание, что перспективный бюджетный план составляется по укрупненным статьям, а также ввиду ограниченности во временных ресурсах для проведения исследования, в рамках данной работы было принято решение рассматривать лишь укрупненные статьи бюджета. В соответствии с действующей системой бюджетной классификации Российской Федерации, крайней точкой детализации был принят порог до подгруппы и ПРз включительно для доходных и расходных статей бюджета соответственно.

3. Третьим этапом проведения прогноза является определение горизонта прогнозирования. Применительно к перспективному финансовому плану, срок его составления установлен в Бюджетном кодексе Российской Федерации и составляет три финансовых года, начиная с года, на который составляется бюджет. В рамках данной работы, ввиду ограниченности во временных ресурсах, горизонт прогнозирования был сужен до одного финансового года.

4. Четвертым этапом проведения прогноза является анализ исторических данных. В данном случае прогнозы разрабатываются на основании отчетов об исполнении бюджета в разрезе статей бюджетной классификации Российской Федерации. Применительно к прогнозированию перспективного финансового плана, анализ исторических данных включает в себя мониторинг, сортировку и организацию бюджетных статей в соответствии со списком прогнозируемых бюджетных статей и горизонтом прогнозирования, определенных на предыдущих этапах. Источником исторических данных для проведения прогноза послужили отчеты об исполнении бюджетов Российской Федерации, размещенные на официальном сайте Федерального Казначейства Российской Федерации.

5. Пятым этапом проведения прогноза является выбор методов прогнозирования. Ввиду ограниченности во временных и материальных ресурсах в рамках данной работы было принято решение использовать лишь некоторые группы методов прогнозирования, а именно математические методы, методы эвристического прогнозирования, а также методы индивидуальных экспертных оценок. В пределах вышеуказанных групп выбор производился на основании комбинирования результатов третьего и четвертого этапов и сопоставимости методов и таких факторов прогнозирования, как горизонт прогнозирования, доступность исторических данных, тренд и цикличность.

6. Шестым этапом проведения прогноза является оценка выбранных методов прогнозирования. Так как интуитивные методы прогнозирования не имеют четких алгоритмов оценки точности, данную оценку возможно провести только для формализованных методов. С точки зрения прогнозирования показателей перспективного финансового плана данный этап представляет собой сравнение полученных прогнозных значений бюджетных статей с фактическими значениями этих статей, с одной стороны (т.е. с данными отчетов об исполнении бюджетов), и с будущими значениями - с другой стороны. В рамках данной работы в качестве количественной оценки точности использовался 
показатель коэффициента детерминации $\mathrm{R}^{2}$.

7. Заключительным этапом проведения прогноза является подготовка прогноза. На данном этапе предполагается использование программных средств статистического прогнозирования.

Для оценки предложенного алгоритма было проведено прогнозирование перспективного финансового плана Иркутской области на 2011 год. Рассмотрим все этапы построения прогноза.

В соответствии с разработанной системой, целью прогнозирования является создание прогноза показателей перспективного финансового плана Иркутской области для поддержки принятия управленческих решений, связанных с тенденциями развития экономики и социальной сферы региона. Объектом прогнозирования служат статьи перспективного финансового плана Иркутской области, в соответствии с фактически используемыми бюджетными статьями.

Горизонт прогнозирования, в рамках данной работы, был принят равным одному финансовому году, который длится с 1 января по 31 декабря 2011 года. Данный горизонт прогнозирования был выбран исходя из доступности исторических данных на момент окончания исследовательской работы, т.е. только до декабря 2010 года.

Источником исторических данных для проведения прогноза послужили отчеты об исполнении бюджетов субъектов Российской Федерации, размещенные на официальном сайте Федерального Казначейства Российской Федерации (Федеральное Казначейство РФ, 2011). Исходные данные были переработаны и представлены в виде таблицы с помесячной разбивкой за период с января 2006 года по декабрь 2010 года: таким образом, число анализируемых периодов составляет 60 месяцев.

Ввиду ограниченности во временных и материальных ресурсах в рамках данной работы было принято решение использовать две основные группы методов прогнозирования - это математические методы прогнозной экстраполяции и экспертные оценки. В пределах данных групп выбор производился на основании сопоставимости методов и факторов прогнозирования. При выборе методов для прогнозирования каждой статьи использовались следующие факторы и методы их оценки:

1. Горизонт прогнозирования - на предыдущем шаге горизонт прогнозирования был определен продолжительностью в один календарный год, что относится к краткосрочному периоду прогнозирования. Таким образом, возможно использовать практически полный арсенал методов математического прогнозирования.

2. Доступность исторических данных - определялась количеством наблюдений (т.е. значений, отличных от нуля) для различных бюджетных статей. Для большинства статей фактические значения колеблются в пределах от 23 до 60 наблюдений. Таким образом, исторические данные по большинству бюджетных статей включают в себя временной промежуток протяженностью от двух до пяти лет, что является достаточным для большей части методов математического прогнозирования.

3. Тренд - определялся на основании графического анализа диаграмм исходных данных с наложенной функцией скользящего среднего с интервалом в 12 значений. Исходя из проведенного анализа диаграмм, можно сделать вывод, что для большинства рассматриваемых бюджетных статей характерен слабый положительный или отрицательный тренд.

4. Цикличность - определялась на основании автокорреляционного анализа и графического анализа автокоррелограмм прогнозируемых бюджетных статей. Анализ приведенных автокоррелограмм свидетельствует, что для большинства рассматриваемых бюджетных статей характерна цикличность с длиной цикла в один календарный год.

В результате оценки вышеприведенных факторов были выбраны следующие методы для прогнозирования бюджетных статей: 
Таблица 1

\begin{tabular}{|c|c|c|}
\hline $\begin{array}{c}\text { Метод } \\
\text { прогнозирования }\end{array}$ & Группа методов прогнозирования & $\begin{array}{c}\text { Кол-во } \\
\text { прогнозируемых } \\
\text { статей }\end{array}$ \\
\hline AРИМА (ARIMA) & Авторегрессионные модели & \multirow{5}{*}{67} \\
\hline CensusII X-11 & \multirow{2}{*}{ Методы декомпозиции } & \\
\hline Декомпозиция (ARIMA) & & \\
\hline $\begin{array}{l}\text { Экспоненциальное } \\
\text { сглаживание Уинтерса }\end{array}$ & $\begin{array}{l}\text { Методы экспоненциального } \\
\text { сглаживания }\end{array}$ & \\
\hline Линейная регрессия & Регрессионные методы & \\
\hline Интервью & Индивидуальные экспертные оценки & 26 \\
\hline
\end{tabular}

Таким образом, формализованные методы прогнозирования охватывают 67 статей из 93 (без учета промежуточных статей), что составляет около $70 \%$.

Так как интуитивные методы прогнозирования не имеют четких алгоритмов оценки точности, данная оценка проводилась только для формализованных методов. Распределение методов с наибольшими показателями коэффициента детерминации приведено в следующей таблице:

Таблица 2

\begin{tabular}{|l|c|c|}
\hline \multicolumn{1}{|c|}{ Метод прогнозирования } & $\begin{array}{c}\text { Кол-во } \\
\text { прогнозируемых } \\
\text { статей }\end{array}$ & \% от общего \\
\hline ARIMA & 0 & 0 \\
\hline CensusII X-11 & 56 & 84 \\
\hline Декомпозиция (ARIMA) & 11 & 16 \\
\hline Метод Уинтерса & 0 & 0 \\
\hline Линейная регрессия & 0 & 0 \\
\hline
\end{tabular}

Таким образом, для большей части всех прогнозируемых бюджетных статей оптимальными методами прогнозирования являются CensusII X-11 и Декомпозиция (ARIMA). Подобные результаты можно объяснить двумя факторами:

1. Метод CensusII X-11 является не столько формализованным методом прогнозирования, сколько набором многочисленных специально разработанных приемов, доказавших свою работоспособность в многолетней практике решения реальных задач в области прогнозирования социальных процессов (StatSoft Inc., 2011]). Поскольку бюджетная система отражает реальные социально-экономические процессы, происходящие в обществе, метод CensusII X-11 дает очень хорошие результаты при прогнозировании бюджетных статей.

2. Методы CensusII X-11 и Декомпозиция (ARIMA) относятся к группе методов декомпозиции, которые являются наиболее применимыми при прогнозировании данных, имеющим сезонный, циклический и трендовый компоненты. В то же время вышеуказанные компоненты присутствуют практически во всех рассматриваемых бюджетных статьях.

Между тем стоит отметить, что для многих прогнозируемых бюджетных статей количественные оценки точности методов ARIMA и экспоненциального сглаживания Уинтерса минорно отличаются от аналогичных оценок метода CensusII X-11, что позволяет использовать их либо в качестве альтернативных методов прогнозирования, либо в качестве составных методов при комплексном прогнозировании.

Распределение значений коэффициента детерминации в рамках выбранных методов для прогнозируемых статей приведено в следующей таблице: 
Таблица 3

\begin{tabular}{|l|c|c|}
\hline Значение коэффициента детерминации, \% & $\begin{array}{c}\text { Кол-во } \\
\text { прогнозируемых } \\
\text { статей }\end{array}$ & \% от общего \\
\hline От 90 до 100 & 16 & 24 \\
\hline От 80 до 89 & 31 & 46 \\
\hline От 70 до 79 & 10 & 15 \\
\hline От 60 до 69 & 3 & 6 \\
\hline От 50 до 59 & 4 & 0 \\
\hline От 40 до 49 & 0 & 4 \\
\hline От 30 до 39 & 3 & 6 \\
\hline
\end{tabular}

Таким образом, для 70\% прогнозируемых статей, точность выбранных методов прогнозирования превышает порог в $80 \%$, что позволяет сделать достаточно реалистичный прогноз на 2011 год.

В рамках данной работы в качестве технического средства реализации процесса прогнозирования для формализованных методов использовалось приложение Microsoft Excel 2007 с установленным комплексом программных средств ForecastX, разработанным компанией John Gault Solutions Inc., Австралия. Прогнозирование с помощью экспертных оценок производилось с использованием упрощенного варианта метода экстраполяции трендов - прогнозное значение для бюджетных статей принималось равным значению предыдущего периода (2010 года), скорректированному с поправкой на коэффициент предполагаемого изменения статей, спрогнозированных с помощью методов формализованного прогнозирования. Так, для доходных статей данный коэффициент составляет $12 \%$, для расходных - 30\%.

В результате проведения прогноза основные показатели перспективного финансового плана Иркутской области на 2011 год имеют следующие значения: прогнозируемые совокупные доходы региона - 93,5 млрд руб., прогнозируемые совокупные расходы региона - 90,5 млрд руб., прогнозируемый профицит бюджета - 3 млрд руб. Расшифровка прогноза в помесячной разбивке приведена в следующей таблице (все данные приведены в миллиардах рублей):

Таблица 4

\begin{tabular}{|c|c|c|c|c|c|c|c|c|c|c|c|c|}
\hline Наименование показателя & 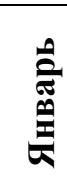 & 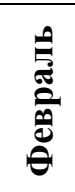 & $\frac{\hbar}{\bar{a}}$ & 离 & 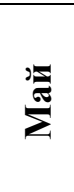 & $\hat{\theta}$ & $\stackrel{\hat{s}}{\underline{3}}$ & 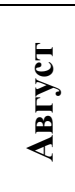 & & & 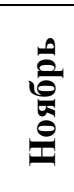 & 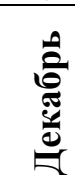 \\
\hline \multicolumn{13}{|l|}{$\begin{array}{l}\text { Раздел 1. Доходы, в млрд } \\
\text { руб. }\end{array}$} \\
\hline $\begin{array}{l}\text { НАЛОГОВЫЕ И } \\
\text { НЕНАЛОГОВЫЕ } \\
\text { ДОХОДЫ }\end{array}$ & 2,9 & 3,1 & 6,6 & 7,5 & 3,5 & 4,4 & 6,0 & 4,1 & 4,2 & 5,2 & 4,4 & 5,8 \\
\hline $\begin{array}{l}\text { БЕЗВОЗМЕЗДНЫЕ } \\
\text { ПОСТУПЛЕНИЯ }\end{array}$ & 3,8 & 3,5 & 2,0 & 3,0 & 3,0 & 2,8 & 3,8 & 3,6 & 2,0 & 3,0 & 3,0 & 2,8 \\
\hline ИТОГО ДОХОДОВ & 6,6 & 6,7 & 8,6 & $\mathbf{1 0 , 5}$ & 6,4 & 7,3 & $\mathbf{9 , 8}$ & 7,7 & 6,2 & 8,2 & 7,4 & 8,6 \\
\hline \multicolumn{13}{|l|}{$\begin{array}{l}\text { Раздел 2. Расходы, в млрд } \\
\text { руб. }\end{array}$} \\
\hline $\begin{array}{l}\text { ОБЩЕГОСУДАРСТВЕНН } \\
\text { ЫЕ ВОПРОСЫ }\end{array}$ & 0,1 & 0,2 & 0,2 & 0,3 & 0,2 & 0,2 & 0,3 & 0,3 & 0,2 & 0,2 & 0,2 & 0,5 \\
\hline $\begin{array}{l}\text { НАЦИОНАЛЬНАЯ } \\
\text { ОБОРОНА }\end{array}$ & 0,0 & 0,0 & 0,0 & 0,0 & 0,0 & 0,0 & 0,0 & 0,0 & 0,0 & 0,0 & 0,0 & 0,0 \\
\hline
\end{tabular}




\begin{tabular}{|c|c|c|c|c|c|c|c|c|c|c|c|c|}
\hline Наименование показателя & 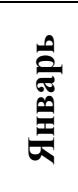 & 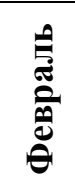 & $\sum_{\bar{\Sigma}}^{\bar{\sigma}}$ & 氨 & 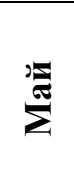 & $\hat{\theta}$ & $\stackrel{0}{\hat{s}}$ & 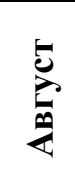 & 言 & tô. & 言 & 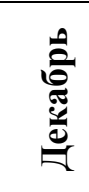 \\
\hline $\begin{array}{l}\text { НАЦИОНАЛЬНАЯ } \\
\text { БЕЗОПАСНОСТЬ И } \\
\text { ПРАВООХРАНИТЕЛЬНАЯ } \\
\text { ДЕЯТЕЛЬНОСТЬ }\end{array}$ & 0,1 & 0,4 & 0,3 & 0,3 & 0,3 & 0,4 & 0,3 & 0,3 & 0,3 & 0,3 & 0,3 & 0,4 \\
\hline $\begin{array}{l}\text { НАЦИОНАЛЬНАЯ } \\
\text { ЭКОНОМИКА }\end{array}$ & 0,2 & 0,2 & 0,3 & 0,5 & 0,4 & 0,4 & 0,4 & 0,4 & 0,4 & 0,4 & 0,4 & 1,0 \\
\hline $\begin{array}{l}\text { ЖИЛИЩНО- } \\
\text { КОММУНАЛЬНОЕ } \\
\text { ХОЗЯЙСТВО }\end{array}$ & 0,0 & 0,0 & 0,0 & 0,1 & 0,0 & 0,0 & 0,1 & 0,1 & 0,1 & 0,0 & 0,1 & 0,2 \\
\hline $\begin{array}{l}\text { ОХРАНА ОКРУЖАЮЩЕЙ } \\
\text { СРЕДЫ }\end{array}$ & 0,0 & 0,0 & 0,0 & 0,0 & 0,0 & 0,0 & 0,0 & 0,0 & 0,0 & 0,0 & 0,0 & 0,0 \\
\hline DВАНИЕ & 0 & 0,4 & 0,5 & 0 , & 0,5 & 0,9 & 0,6 &, 3 & 0,5 & 0 & 0,5 & 1,0 \\
\hline $\begin{array}{l}\text { КУЛЬТУРА, } \\
\text { КИНЕМАТОГРАФИЯ, } \\
\text { СРЕДСТВА МАССОВОЙ } \\
\text { ИНФОРМАЦИИ }\end{array}$ & 0,1 & 0,1 & 0,1 & 0,2 & 0,2 & 0,2 & 0,2 & 0,2 & 0,2 & 0,2 & 0,2 & 0,5 \\
\hline $\begin{array}{l}\text { ЗДРАВООХРАНЕНИЕ, } \\
\text { ФИЗИЧЕСКАЯ КУЛЬТУРА } \\
\text { И СПОРТ } \\
\end{array}$ & 0,2 & 0,4 & 0,4 & 0,5 & 0,4 & 0 , & 0,5 & 0,5 & 0,4 & 0,5 & 0,5 & 0,8 \\
\hline $\begin{array}{l}\text { СОЦИАЛЬНАЯ } \\
\text { ПОЛИТИКА }\end{array}$ & 1,5 & 1,6 & 1,8 & 2,2 & 1,6 & 1,7 & 1,7 & 2,1 & 1,5 & 1,7 & 1,6 & 2,2 \\
\hline $\begin{array}{l}\text { МЕЖБЮДЖЕТНЫЕ } \\
\text { ТРАНСФЕРТЫ }\end{array}$ & 3,0 & 3,7 & 3,0 & 3,4 & 3,3 & 4,7 & 2,8 & 3,2 & 3,4 & 4,2 & 3,1 & 4,4 \\
\hline ИТОГО РАСХОДОВ & 5,4 & 6,9 & 6,7 & 8,0 & 7,0 & 9,1 & 6,9 & 7,4 & 7,0 & 8,0 & 7,0 & 11,0 \\
\hline
\end{tabular}

Вместе с тем наряду с ретроспективной оценкой точности предлагаемых методов прогнозирования важную роль также играет перспективная оценка. В соответствии со справкой Министерства финансов Иркутской области об исполнении консолидированного бюджета Иркутской области по состоянию на 1 июля 2011 года, исполнение бюджета по доходам составляет 46,4 млрд руб., по расходам - 31,9 млрд руб. В соответствии с представленным прогнозом, доходная часть составляет 46,1 млрд руб., расходная - 43 млрд руб. Таким образом, расхождение прогноза и факта по доходам составляет меньше $1 \%$, по расходам - около 30\%. То есть можно сделать вывод, что предлагаемая система прогнозирования отдельных показателей перспективного финансового плана имеет высокую точность в прогнозировании доходов и среднюю - в прогнозировании расходов. Последняя проблема может быть решена в результате доработки методов прогнозирования расходных статей путем комбинирования.

\section{Список литературы}

1. Бестужев-Лада И.В. Рабочая книга по прогнозированию. М.: Мысль, 1982.

2. Бюджетный кодекс Российской Федерации: Федер. закон от 31 июля 1998 г. № 145Ф3. М.: Финансы и статистика, 2002.

3. Гранберг А.Г. Статистическое моделирование и прогнозирование: Учебн. пособие / под. ред. А.Г. Гранберга. М.: Финансы и статистика, 1990.

4. Отчетность об исполнении бюджетов субъектов РФ и местных бюджетов [Электронный peсурс] / Федеральное Казначейство PФ. URL: http://www.roskazna.ru/reports/mb.html (дата посещения: 30.03.2011).

5. Информационно-аналитический комплекс «Бюджетная система РФ» [Электронный ресурс] / AHO Центр информационных исследований. URL: 
http://www.budgetrf.ru/Publications/Education/distant/publfinance_2003_/publfinance_200 3_02_/publfinance_2003_02_000.htm (дата посещения: 15.02.2010).

6. Мысляева И.М. Государственные и муниципальные финансы: Учебник. Изд. 2-е, перераб. и дон. М: ИНФРА-М, 2007.

7. Советник по прогнозированию: Электронный учебник StatSoft [Электронный ресурс] / StatSoft Inc.

URL:

http://www.statsoft.ru/home/portal/applications/ForecastingAdvisor/Methods/CensusII/cens usII.htm (21 февраля 2011).

8. Holton Wilson, J. (2002), Business forecasting, Australia, McGraw-Hill Higher Education. 